\title{
Smart Traffic Management System
}

\author{
${ }^{1}$ Ninad Lanke \\ B.E IT (pursuing) \\ 202, Shrikrishna soc, Sahakarnagar no-2, \\ Pune-411009, India
}

\author{
${ }^{2}$ Sheetal Koul \\ M.E (Computer Networks)-pursuing \\ Department of Information Technology, \\ SKNCOE, Pune-411041
}

\begin{abstract}
Traffic congestion is a major problem in many cities of India along with other countries. Failure of signals, poor law enforcement and bad traffic management has lead to traffic congestion. One of the major problems with Indian cities is that the existing infrastructure cannot be expanded more, and thus the only option available is better management of the traffic. Traffic congestion has a negative impact on economy, the environment and the overall quality of life. Hence it is high time to effectively manage the traffic congestion problem. There are various methods available for traffic management such as video data analysis, infrared sensors, inductive loop detection, wireless sensor network, etc. All these methods are effective methods of smart traffic management. But the problem with these systems is that the installation time, the cost incurred for the installation and maintenance of the system is very high. Hence a new technology called Radio Frequency Identification (RFID) is introduced which can be coupled with the existing signaling system that can act as a key to smart traffic management in real time. This new technology which will require less time for installation with lesser costs as compared to other methods of traffic congestion management. Use of this new technology will lead to reduced traffic congestion. Bottlenecks will be detected early and hence early preventive measures can be taken thus saving time and money of the driver.
\end{abstract}

\section{Keywords}

\section{RFID, GSM, Traffic congestion.}

\section{INTRODUCTION}

Traffic congestion on road networks is nothing but slower speeds, increased trip time and increased queuing of the vehicles. When the number of vehicles exceeds the capacity of the road, traffic congestion occurs. In the metropolitan cities of India traffic congestion is a major problem. Traffic congestion is caused when the demand exceeds the available road capacity. This is known as saturation [1]. Individual incidents such as accidents or sudden braking of a car in a smooth flow of heavy traffic have rippling effects and cause traffic jams ${ }^{[2]}$. There are even severe security problems in traffic system due to anti social elements which also leads to stagnation of traffic at one place. In country like India, there is an annual loss of Rs 60,000 crores due to congestion (including fuel wastage). Congestion in India has also led to slow speeds of freight vehicles, and increased waiting time at checkpoints and toll plazas ${ }^{[3]}$. The average speed of vehicles on key corridors like Mumbai-Chennai, Delhi-Chennai is less than $20 \mathrm{kmph}$, while it is mere $21.35 \mathrm{kmph}$ on Delhi-Mumbai stretch. As per the transport corporation of India and IIM, India's freight volume is increasing annually at a rate of $9.08 \%$ and that of vehicles at $10.76 \%$, but that of road is only by $4.01 \%$. This has resulted in reduced road space in accordance with the number of total vehicles ${ }^{[3]}$.The average fuel mileage in India is only $3.96 \mathrm{kmpl}$. The major reason for this is traffic congestion ${ }^{[3]}$.India is the $2^{\text {nd }}$ most populated country after China in Asia, thus with increase in population, the number of vehicles also increase ${ }^{[4]}$.The economic growth has certainly has had an impact on urban traffic. As the income rises, more and more people begin to go for cars rather than two wheelers ${ }^{[5]}$.Hence there is a need to manage traffic in a smart way as the management of traffic with the conventional way such as the signaling system is not having a major effect in curbing congestion of vehicular traffic.

\section{EXISTING METHODOLOGY}

\subsection{Inductive Loop Detection}

Inductive loop detection works on the principle that one or more turns of insulated wire are placed in a shallow cutout in the roadway, a lead in wire runs from roadside pull box to the controller and to the electronic unit located in the controller cabinet. When a vehicle passes over the loop or stops, the induction of the wire is changed. Due to change in induction, there is change in the frequency. This change in the frequency causes the electronic unit to send a signal to the controller; indicating presence of the vehicle ${ }^{[6]}$.Inductive loop detection is useful in knowing the vehicle presence, passage, occupancy and even the number of vehicles passing through a particular area ${ }^{[6,7]}$. But there are few problems with this system. These include poor reliability due to improper connections made in the pull boxes and due to application of sealant over the cutout of the road. If this system is implemented in poor pavement or where digging of the roads is frequent then the problem of reliability is aggravated ${ }^{[4,5,8]}$.

\subsection{Video Analysis}

Video analysis consists of a smart camera placed which consists of sensors, a processing unit and a communication unit ${ }^{[9]}$. The traffic is continuously monitored using a smart camera. The video captured is then compressed so as to reduce the transmission bandwidth. The video analysis abstracts scene description from the raw video data. This description is then used to compute traffic statistics. This statistic includes frequency of the vehicles, average speed of the vehicles as well as the lane occupancy ${ }^{[9,10]}$.The problems associated with video analysis are - (a) the overall cost of the system is quite high (b) the system gets affected in case of heavy fog or rains (c) night time surveillance requires proper street lighting ${ }^{[6,8]}$. 


\subsection{Infrared Sensors}

Infrared sensors are used to detect energy emitted from vehicles, road surfaces and other objects. The energy captured by these infrared sensors is focused onto an infrared sensitive material using an optical system which then converts the energy into the electric signals. These signals are mounted overhead to view the traffic. Infrared sensors are used for signal control, detection of pedestrians in crosswalks and transmission of traffic information [11]. The basic disadvantages of infrared sensors are that the operation of the system may be affected due to fog; also installation and maintenance of the system is tedious ${ }^{[5,8]}$.

\section{SMART TRAFFIC MANAGEMENT SYSTEM}

\subsection{Background}

A Radio Frequency Identification (RFID) system consists of RFID controller and RFID tag.

\section{1) RFID Controller:}

The RFID controller consists of RFID interrogator. This interrogator is used for the communication with the RFID tag. The RFID controller then gets the signals/data received by the interrogator. Messaging interference is used to send commands and data messages from the controller components. Controller core is present inside the RFID controller. The controller core listens to the interrogators and depending upon the configuration; the controller core can perform read/write operations upon the RFID tag or can do both listening and performing operations ${ }^{[5]}$.The RFID controller can have serial interface through which external GSM/GPRS devices can be interfaced with it to make a dual radio device.

\section{2) RFID Tag:}

RFID tags are wireless devices which make use of radio frequency electromagnetic fields to transfer data, which is used for identifying and tracking of the objects. RFID tags are of two types: Active and Passive ${ }^{[12]}$. Active RFID has a battery installed, which the passive RFID doesn't have. Passive RFID has to depend on external source for working. Tags information can be stored in a non-volatile memory. Tag consists of a Radio Frequency transmitter and receiver. Each tag can be assigned a unique serial number ${ }^{[13]}$.

\subsection{Relevant Algorithm}

\section{Input:}

Max_red denotes the maximum time for which the signal can be red.

Max_green denotes the maximum time for which the signal can be green.

Min_freq_count denotes the minimum frequency of vehicles passing per second stored statically in controllers.

Act_freq_count denotes the actual frequency of the vehicles passing per second $=\sum$ vehicles/second.
Timer denotes the actual timer count.

\section{Algorithm:}

1. When the signal turn green.

While (Timer $<$ Max_green and Timer is not 0 ) do

If (Act_freq_count>Min_freq_count)

Keep the signal green.

Decrement timer count by 1 .

Else if (Act_freq_count $<=$ Min_freq_count)

Goto 2 .

End

2. Make the signal red. Turn the adjacent signal green. Goto 1 .

Desired Output: Effective congestion management

\subsection{System Overview}

Each vehicle can be installed with a RFID tag. This RFID tag would store all the information regarding the vehicle such as the vehicle number, etc. RFID tags can be used in identifying each vehicle uniquely and also help the driver to receive some traffic messages. The existing signaling system can be coupled with the RFID controller. As described in figure 1, each signal can have the information regarding every vehicle that passes by it. Thus when a vehicle passes by a signal, the signal can automatically keep the count of the vehicles passing by it, and help in detection of traffic congestion. Each signal should be stored with a threshold value for which it should be red and green. Now depending upon the frequency of the vehicles passing by the signal per second, the timer can be dynamically controlled. Each controller of the signal should be stored with a value of minimum frequency of the vehicles passing by the signal. As soon as this minimum frequency is reached, the controller should send a command to the signal to turn red. Thus the signal is controlled dynamically. For example, suppose for a signal, maximum time for which a signal can be red is set to be 30 seconds and maximum time for which the signal can be green is set as 20 seconds. The controller is stored with the value of minimum frequency of vehicles passing by it per second as 5 . Now suppose the signal turns green, the timer starts with a maximum value of 20 . Initially the frequency of the vehicles passing the signal per second is 10 , after 10 seconds this frequency reduces to 5 , and then automatically the RFID controller sends a command to the signal to turn red. Thus the signal turns red and its adjacent signal in that junction turns green. This process continues in a cycle. Thus dynamic controlling of the signal helps in reducing the wastage of time. This also helps in avoiding traffic congestion as priority is given to a high vehicular traffic road. This system helps in detection of traffic congestion. If the frequency of the vehicles passing the signal per second remains higher than the value set even though 


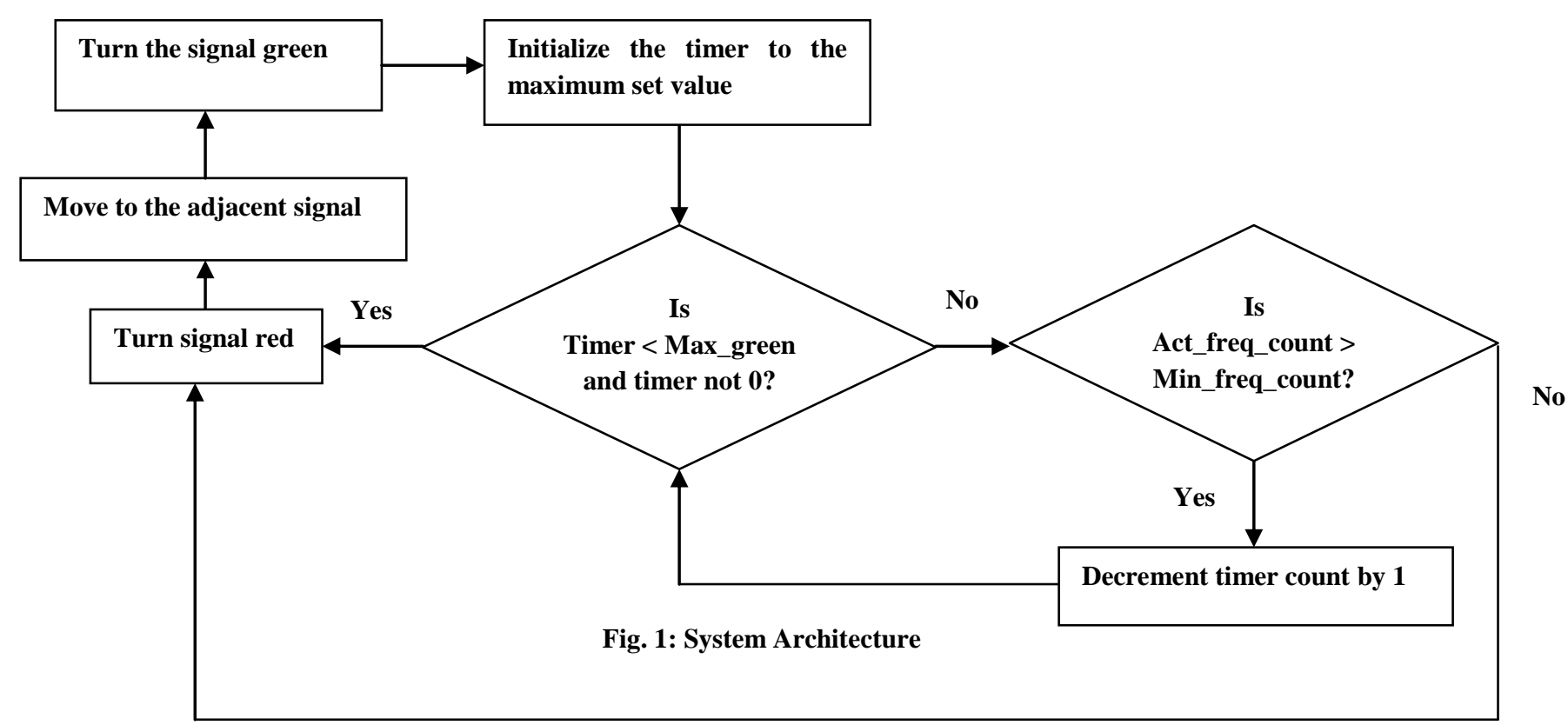

the maximum value of the timer is reached, then the congestion has occurred at that point. Once the congestion has been detected, the RFID controller can send a message to its preceding signal's controller notifying it to temporarily stop traffic along that stretch. After receiving the message from its successor signal the RFID controller will put ON the red signal for that stretch towards that congested crossing point for a predefined time period. When the congestion is released at the crossing, the respective signal's controller will send another message to its earlier controller indicating to resume the traffic flow again in that direction. Accepting this message the controller of the preceding signal put the red light OFF and green signal $\mathrm{ON}$ and restart the signal cycle as before.

\section{APPLICATIONS}

\subsection{Detection and Management of traffic Congestion}

In addition to the earlier method of traffic congestion detection, one more method can be used. A server can be maintained which can receive certain crucial data calculated by the Controller of the signals. The main aim is to implement a system that would trace the travel time of individual cars as they pass the roadside controllers and compute an average trip time using a rule-based system to decide whether the area is congested or uncongested. If congestion is sensed then system would control traffic signals / generate automatic re-routing messages to selected approaching vehicles.

\subsection{Automatic detection of speed limit Violation}

We can use this technique to calculate the speed of a motorist and to detect if he violates the prescribed/set speed limit. If the motorist violates the rule, a warning message will be sent to the motorist via audio and/or video interface and penalty will be calculated in the server and billed monthly to the vehicle owner ${ }^{[14]}$.

\subsection{Automatic Billing of Core Area / Toll Charges}

Automatic toll collection and automatic "core area charge" collections are also done using the same framework. Controller unit will be placed at toll-booth and along the motor able roads around the core area which will detect each individual vehicle uniquely within its zone by capturing their device ids and will keep records of the time during which the vehicle was seen by those Controllers within its reading zone. This information will be sent to a main server. Accordingly the main server will calculate the charges and raise bills against the vehicle ids ${ }^{[14]}$.

\section{CONCLUSION AND FUTURE WORK}

The proposed work focuses on Smart Traffic management System using RFID which will eliminate the drawbacks of the existing system such as high implementation cost, dependency on the environmental conditions, etc. The proposed system aims at effective management of traffic congestion. It is also cost effective than the existing system.

Furthermore, the study presents the problems in metropolitan areas all over the world caused by congestions and the related sources. Congestions developed to a problem, which affects economies worldwide. Particularly metropolitan areas are worst hit under these conditions. Congestions have a negative impact on the financial situation of a country, on the environment and hence the overall quality of life. The proposed system can be enhanced by using any other powerful communication network other than GSM.

\section{REFERENCES}

[1] $21^{\text {st }}$ Century operations Using $21^{\text {st }}$ Century Technologies. U.S Department Of transportation Federal Highway Administration.2008-08-29. Retrieved 2008-09-25. http://www.ops.fhwa.dot.gov/aboutus/opstory.htm

[2] William Beaty. Jan 1998. Traffic Waves "Sometimes one driver can vastly improve traffic". http://www.amasci.com/amateur/traffic/traffic1.html 
[3] Dipak K Dash, TNN May 31, 2012. "India loses Rs 60,000 crore due to traffic congestion: Study". Times Of India. http://articles.timesofindia.indiatimes.com/201205-31/india/31920307_1_toll-plazas-road-spacestoppage

[4] Azeem Uddin, Draft, 23 March 2009. Traffic congestion in Indian cities: Challenges of a Rising power. http://www.visionwebsite.eu/UserFiles/File/filedascarica re/Scientifci\%20Partners,Papers\%28Kyoto\%29/Draft_ko c_Azeem\%20Uddin.pdf

[5] FHWA-HRT-06-108. October 2006. Traffic Detector Handbook: Third Edition-Volume I. http://www.fhwa.dot.gov/publications/research/operation s/its/06108/

[6] US7245220 B2. Jul 17, 2007. Radio frequency identification (RFID) controller. http://www.google.com/patents/US7245220

[7] Ali, S.S.M.Indian Inst. of Technol. Madras, Chennai, India, George, B.; Vanajakshi L.: A simple multiple loop sensor configuration for vehicle detection in an undisciplined traffic Sensing Technology (ICST), 2011 Fifth International Conference21568065. http://ieeexplore.ieee.org/xpl/articleDetails.jsp?tp=\&arnu mber $=6137062 \& u r l=h t t p \% 3 \mathrm{~A} \% 2 \mathrm{~F} \% 2$ Fieeexplore.ieee.or g\%2Fxpls\%2Fabs_all.jsp\%3Farnumber\%3D6137062

[8] FHWA-RD-96-100. July 1995. Detection Technology: IVHSVolume-

1.http://ntl.bts.gov/DOCS/96100/index.html

[9] Bing-Fei Wu. Dept of Electr. And control eng, Nat. Chiao Tung Univ, Hsinchu, Taiwan. A new Approach to Video-based Traffic surveillance using fuzzy hybrid Information Inference Mechanism.March 2013.IEEE IntelligentTraffic
http://ieeexplore.ieee.org/xpl/articleDetails.jsp?arnumber $=6264098$

[10] Xianbin Cao ; Sch. of Electron. \& Inf. Eng., Beihang Univ., Beijing, China ; Changxia Wu ; Jinhe Lan ; Pingkun Yan : Vehicle Detection and Motion Analysis in Low-Altitude Airborne Video Under Urban Environment Circuits and Systems for Video Technology, IEEE Transactions on (Volume:21 , Issue: 10 ) 10518215http://ieeexplore.ieee.org/xp1/login.jsp?tp=\&arnumb er=5955106\&url=http\%3A\%2F\%2Fieeexplore.ieee.org \%2Fxpls\%2Fabs_all.jsp\%3Farnumber\%3D5955106

[11] Hussain, T.M. ; Dept. of Electr. Eng., City Univ. of New York, NY, USA ; Saadawi, T.N. ; Ahmed, S.A. Overhead infrared sensor for monitoring vehicular traffic:Vehicular Technology, IEEE Transactions on (Volume:42, Issue: 4 ) 0018-9545

http://ieeexplore.ieee.org/xpl/login.jsp?tp=\&arnumber=26076 $4 \&$ url=http $\% 3 \mathrm{~A} \% 2 \mathrm{~F} \% 2$ Fieeexplore.ieee.org $\% 2 \mathrm{Fxpls} \% 2$ Fabs_all.jsp\%3Farnumber\%3D260764

[12] Bichlien Hoang,Ashley Caudill: EEE Emerging Technology portal, 2012 http://www.ieee.org/about/technologies/emerging/rfid.pd $\mathrm{f}$

[13]http://en.wikipedia.org/wiki/Radiofrequency_identification

[14] Wang Hongjian ; Chongqing Special Equip. Quality Safe Inspection Center, Chongqing, China ; Tang Yuelin ; Li Zhi RFID Technology Applied in Highway Traffic Management 978-1-4244-8683-0. Optoelectronics and Image Processing (ICOIP), 2010 International Conference on (Volume: http://ieeexplore.ieee.org/xpl/login.jsp?tp=\&arnumber $=5$ 663108\&url=http\%3A\%2F\%2Fieeexplore.iee.org\%2Fx pls\%2Fabs_all.jsp\%3Farnumber\%3D5663108 